\title{
DetlefSack Hrsg.
}

Wirtschaftskammern im

europäischen Vergleich

SpringerVS 
Wirtschaftskammern im europäischen Vergleich 
Detlef Sack

(Hrsg.)

\section{Wirtschaftskammern im europäischen Vergleich}

黑 SpringerVS 
Herausgeber

Detlef Sack

Fakultät für Soziologie

Universität Bielefeld

Bielefeld, Deutschland

ISBN 978-3-658-16933-6

ISBN 978-3-658-16934-3 (eBook)

DOI 10.1007/978-3-658-16934-3

Die Deutsche Nationalbibliothek verzeichnet diese Publikation in der Deutschen Nationalbibliografie; detaillierte bibliografische Daten sind im Internet über http://dnb.d-nb.de abrufbar.

\section{Springer VS}

(C) Springer Fachmedien Wiesbaden GmbH 2017

Das Werk einschließlich aller seiner Teile ist urheberrechtlich geschützt. Jede Verwertung, die nicht ausdrücklich vom Urheberrechtsgesetz zugelassen ist, bedarf der vorherigen Zustimmung des Verlags. Das gilt insbesondere für Vervielfältigungen, Bearbeitungen, Übersetzungen, Mikroverfilmungen und die Einspeicherung und Verarbeitung in elektronischen Systemen.

Die Wiedergabe von Gebrauchsnamen, Handelsnamen, Warenbezeichnungen usw. in diesem Werk berechtigt auch ohne besondere Kennzeichnung nicht zu der Annahme, dass solche Namen im Sinne der Warenzeichen- und Markenschutz-Gesetzgebung als frei zu betrachten wären und daher von jedermann benutzt werden dürften.

Der Verlag, die Autoren und die Herausgeber gehen davon aus, dass die Angaben und Informationen in diesem Werk zum Zeitpunkt der Veröffentlichung vollständig und korrekt sind. Weder der Verlag noch die Autoren oder die Herausgeber übernehmen, ausdrücklich oder implizit, Gewähr für den Inhalt des Werkes, etwaige Fehler oder Äußerungen. Der Verlag bleibt im Hinblick auf geografische Zuordnungen und Gebietsbezeichnungen in veröffentlichten Karten und Institutionsadressen neutral.

Lektorat: Jan Treibel

Gedruckt auf säurefreiem und chlorfrei gebleichtem Papier

Springer VS ist Teil von Springer Nature

Die eingetragene Gesellschaft ist Springer Fachmedien Wiesbaden GmbH

Die Anschrift der Gesellschaft ist: Abraham-Lincoln-Str. 46, 65189 Wiesbaden, Germany 


\section{Inhaltsverzeichnis}

Industrie- und Handelskammern und institutioneller

Wandel - Einleitung . . . . . . . . . . . . . . . . . . . . . . . .

Detlef Sack

Wirtschaftskammern als politische Akteure: Organisation,

Strategie und Einfluss.

Patrick Bernhagen

Stabilität und Wandel der britischen Industrie- und

Handelskammern . .

Robert J. Bennett

Vom lokalen Notablen zur regionalen Verwaltung? -

Institutioneller Wandel der französischen

Chambres de commerce et d'industrie

Christine Quittkat und Detlef Sack

Institutioneller Wandel der spanischen Industrie- und

Handelskammern .

Iván Medina und Joaquim M. Molins

Strukturwandel der ungarischen Handels- und

Industriekammern nach 1989/1990.

Péter Krisztián Zachar

Stabilität und Wandel der polnischen Industrie- und

Handelskammern

Urszula Kurczewska 
Die dänische Handelskammer - Historische Wurzeln und aktuelle Reformen. . . . . . . . . . . . . . . . . . . . . . . . . 183

Karsten Ronit

Institutioneller Wandel der Wirtschaftskammer Österreich . . . . . . . 209

Ulrich E. Zellenberg

Wirtschaftskammer und Korporatismus in Österreich .......... 233

Ferdinand Karlhofer

Funktionale Selbstverwaltung durch die deutschen Industrie- und Handelskammern - Grundkonzeption, Ausgestaltung und Kritik . . . . 257

Winfried Kluth

Protest, Recht und Europäisierung - Stabilität und Wandel der deutschen Industrie- und Handelskammern . . . . . . . . . . . . . . . . 281 Detlef Sack

Die Legitimität heterogener Wirtschaftskammern Berufliche Sozialisation und Selbstverwaltung bei Unternehmern und Handwerkern. . . . . . . . . . . . . . . . . . . . . . . 309

Katharina van Elten und Sebastian Fuchs

Wandel von Policies oder Institutionen? Politische Prozesse und die Rolle von Kammern in der Berufsbildung . . . . . . . . . . . . . . . 337 Janis Vossiek

Institutionen und regionale Innovationsfähigkeit - Die Rolle deutscher Industrie- und Handelskammern . . . . . . . . . . . . . . . . . . 361

Wolfgang Maennig, Michaela Ölschläger und Hans-Jörg Schmidt-Trenz

Kammern und gesundes Arbeiten in kleinen Unternehmen Potenziale und Empirie

Jens Maylandt

Institutioneller Wandel europäischer Chambers of Commerce im Vergleich - Fazit.

Detlef Sack 


\section{Autorenverzeichnis}

Prof. Dr. Robert J. Bennett Department of Geography, University of Cambridge, Cambridge, England

Prof. Dr. Patrick Bernhagen Institut für Sozialwissenschaften, Universität Stuttgart, Stuttgart, Deutschland

Dr. Katharina van Elten Sektion Politikwissenschaft, Ruhr-Universität Bochum, Bochum, Deutschland

Sebastian Fuchs Mannheimer Zentrum für Europäische Sozialforschung, Universität Mannheim, Mannheim, Deutschland

Prof. Dr. Ferdinand Karlhofer Institut für Politikwissenschaft, Innsbruck, Österreich

Prof. Dr. Winfried Kluth Lehrstuhl für Öffentliches Recht, Martin-LutherUniversität, Halle, Deutschland

Dr. Urszula Kurczewska Warsaw School of Economics, University of Warsaw, Warschau, Polen

Prof. Dr. Wolfgang Maennig Wirtschaftspolitik, Universität Hamburg, Hamburg, Deutschland

Dr. Jens Maylandt Sozialforschungsstelle, Technische Universität Dortmund, Dortmund, Deutschland

Prof. Dr. Iván Medina Constitutional Law \& Political Science, Universitat de València, València, Spanien

Prof. Dr. Joaquim M. Molins Unidad de Ciencia Política y Administración Pública, Universitat Autònoma de Barcelona, Barcelona, Spanien 
Dr. Michaela Ölschläger Handelskammer Hamburg, Hamburg, Deutschland

Dr. Christine Quittkat Mannheimer Zentrum für Europäische Sozialforschung, Mannheim, Deutschland

Prof. Dr. Karsten Ronit Faculty of Social Sciences, University of Copenhagen, Kopenhagen, Dänemark

Prof. Dr. Detlef Sack Fakultät für Soziologie, Universität Bielefeld, Bielefeld, Deutschland

Prof. Dr. Hans-Jörg Schmidt-Trenz Handelskammer Hamburg, Hamburg, Deutschland

Dr. Janis Vossiek Fachbereich für Politik- und Verwaltungswissenschaft, Universität Konstanz, Konstanz, Deutschland

Prof. Dr. Péter Krisztián Zachar Department for International Relations and History, Kodolányi János University of Applied Sciences, Budapest, Ungarn

Dr. Ulrich E. Zellenberg Abteilung Recht und Organe, Wirtschaftskammer Österreich, Wien, Österreich 


\title{
Strukturwandel der ungarischen Handels- und Industriekammern nach 1989/1990
}

\author{
Péter Krisztián Zachar
}

\section{$1 \quad$ Einleitung}

Die Kammern des Wirtschaftslebens entstanden in Ungarn in der bürgerlichen Epoche des 19. Jahrhunderts vor allem auf französischen Einfluss und etwas später nach deutschen Vorbildern. ${ }^{1}$ Das Königreich Ungarn lag damit im „Trend der Zeit" und übernahm die sich in westeuropäischen Staaten etablierenden neuen Modelle der funktionalen Selbstverwaltung (Kluth 1997, S. 12) gleich zu Beginn (Korinek 1991; Pelinka und Smekal 1996; Kluth 2005; Gergely 2006; Strausz und Zachar 2008). In der damaligen ungarischen Gesellschaft hatten die Industrie- und Handelskammern, die nach dem Prinzip der allgemeinen und obligatorischen Interessensvertretung organisiert wurden und als eine liberal ausgerichtete Selbstverwaltungsstruktur galten, besonders wichtigen Einfluss auf die Weiterentwicklung des Handels, auf die Durchsetzung der Marktverhaltensregeln, auf die Unterstützung der allgemeinen Interessensdurchsetzung der an der Wirtschaft beteiligten Parteien, auf die Verbreitung neuer Techniken und Leitungsmodelle (Management), sowie auf die Beratung und Meinungsbildung für die öffentliche Verwaltung in Wirtschaftsfragen. Nachdem die tragischen Ereignisse des 20. Jahrhunderts zur Etablierung eines staatssozialistischen, kommunistischen Staatsmodells in Ungarn geführt hatten, wurden die bestehenden bürgerlichen Instanzen

${ }^{1}$ Die vorliegende Studie entstand im Rahmen und mit Unterstützung des János Bolyai Forschungsstipendiums der Ungarischen Akademie der Wissenschaften.

P.K. Zachar $(\bowtie)$

Department for International Relations and History, Kodolányi János University of Applied Sciences, Budapest, Ungarn

E-Mail: zacharpeter@gmail.com 
und damit auch die Kammern obsolet. Mit der politischen Wende 1989/1990 kam es auch zu einer Umstrukturierung der Gesellschafts- und Wirtschaftsverhältnisse in Ungarn, mit der eine Rückkehr zur Marktwirtschaft einherging, was Hoffnungen auf eine Wiederbelegung des traditionsreichen Kammersystems weckte.

Die vergangenen zweieinhalb Jahrzehnte haben jedoch gezeigt, dass die Frage der Chambers of Commerce $(\mathrm{CoC})$ seit der politischen Wende immer wieder umstritten, durch und durch politisch und bis heute nicht geklärt ist. Die Entstehung, parlamentarische Annahme und Inhalte der in den vergangenen zwei Jahrzehnten geschaffenen Kammerrechtsnormen zeigen keine einheitliche Stellungnahme, keinen über Regierungszeiten hinausweisenden Konsens. Es gibt keine gemeinsamen Narrative zum Wirkungsrahmen, zur Rolle und zur Funktion der Kammern in Ungarn. Gemäß den Erwartungen, die man an ein Transformationsland stellen würde, änderte sich die Einstellung der politischen Machthaber zu den Kammern in Ungarn je nachdem, ob mehr oder weniger politische Vorteile aus der Beziehung zu ihnen zu erwarten waren, beziehungsweise welcher politischen Seite man die jeweilige Kammerleitung zuordnete. Dies führte zu immer neuen Modifikationen des Rechtsrahmens und wirft wiederholt Fragen zur Legitimation und Funktionsfähigkeit der Institution auf. All das wirkte sich auf die Kammermitgliedszahlen wie auch auf das Dienstleistungsangebot der CoC nachhaltig aus. Die Machtinhaber nach der Wende waren - kurz gesagt - bestrebt, darauf hinzuwirken, dass die Tätigkeit dieser Organisationen nur formal bleibe und ihre Interessenvertretungsarbeit, die unvermeidlich Konflikte mit der Sphäre der Politik generiert, auf ein Minimum beschränkt werde. Somit waren die $\mathrm{CoC}$ in der gesamten zweiten Hälfte des 20. Jahrhunderts stets ein „Spielball der Politik" (Strausz und Zachar 2010, S. 227): In den Jahrzehnten der kommunistischen Diktatur und des „Staatssozialismus“ war es das eindeutige Ziel der politischen Macht, die CoC aufzulösen beziehungsweise zu einfachen Machtinstrumenten zu degradieren, was zeitweise mit drastischen, zeitweise mit feineren Mitteln erreicht wurde. Es ist aber zu konstatieren, dass sich die Attitüde der politischen Macht zu den autonomen Interessenvertretungsorganisationen selbst nach der politischen Wende 1989/1990 nicht wirklich wandelte und die funktionale Selbstverwaltung gerade von den Machthabern delegitimiert wurde.

Für das vorliegende Kapitel ergeben sich damit einige Forschungshypothesen, die für die interdisziplinäre und vergleichende Analyse der europäischen $\mathrm{CoC}$ neue Erkenntnisse bringen könnten. ${ }^{2}$ Einerseits sehen in Transformationsländern politische Machthaber in den Interessenvertretungen (und funktionalen

${ }^{2}$ Diese Organisationen werden in Ungarn als Handels- und Industriekammern bezeichnet. 
Selbstverwaltungen) eine Konkurrenz, an deren Schwächung sie interessiert sind. Andererseits gibt es immer wieder Perioden, in denen die $\mathrm{CoC}$ ihre Interessen durchsetzen können, aber trotzdem unter dem Mantel der Politik zu verbleiben scheinen. Das hängt mit der internen Struktur, den schwachen Mitgliedszahlen und den Konflikten mit anderen Unternehmerverbänden zusammen. Die Darstellung der ungarischen Verhältnisse trägt damit zur europäischen Debatte über das Wesen und den Strukturwandel der Kammern bei, besonders auch im Hinblick auf die Entwicklungen, die sich in letzter Zeit in Spanien und auch in Polen ergeben haben (Medina und Molins; Kurczewska i. d. B.).

\section{Historische Entwicklung der ungarischen Handels- und Industriekammern}

Die CoC im modernen Sinne sind in Ungarn durch den Gesetzesartikel VI. des Jahres 1868 entstanden. Diese zweite Regelung nach dem früheren provisorischen österreichischen Kammergesetz des Jahres $1850^{3}$ setzte die Kammern auf eine liberalere Grundlage, gewährleistete ihre tatsächliche Autonomie und ihren freien Spielraum, wobei jedoch das Prinzip der allgemeinen und obligatorischen Interessensvertretung erhalten blieb. Gegenüber den stark zentralisierten und bürokratisierten französischen Interessensvertretungsmustern entstanden somit hier - ähnlich den Gebieten des Deutschen Reiches und des Habsburger-Reiches - freiere Kammerstrukturen, die hinsichtlich ihrer Befugnisse über einen breiteren Spielraum verfügten (Kluth und Rieger 2004).

In dieser Epoche waren die Tätigkeitsfelder der Kammern eng mit der Herausbildung der bürgerlichen Gesellschaftsordnung sowie der modernen Wirtschaft verbunden. Durch die Ideen des Liberalismus, der Selbstverwaltung (Lorenz von Stein) und der Interessenvertretung entstand auch ein Bedarf nach den modernen Techniken und Wissensinhalten dieses Wirtschaftslebens. Somit hatten die $\mathrm{CoC}$ eine bedeutende Rolle in der Verbreitung westeuropäischer Muster. So waren sie an der Schaffung eines neuen Industriegesetzes beteiligt, sie sprachen sich für eine moderne Sozialpolitik (günstige Arbeiterwohnungen, Sonntagsruhe, Arbeiterschutz- und Krankenversicherungsgesetze) aus, sie propagierten die Infrastrukturentwicklung (Eisenbahn, Fernmeldeämter, Brücken) und die Weiterbildungsmöglichkeiten für Kaufleute. In der Epoche des entfalteten Kapitalismus

\footnotetext{
${ }^{3} 1850$ wurden in Ungarn und seinen Nebenländern elf Kammern mit Pflichtmitgliedschaft aufgestellt. Sie wurden der Zuständigkeit des Wiener Handelsministeriums unterstellt.
} 
zeichneten sich dadurch im Allgemeinen drei Gebiete der Kammertätigkeit ab: wirtschaftsorganisatorische und Interessenvertretungstätigkeit, Fragen der Fachund Weiterbildung sowie Übernahme von öffentlichen Verwaltungsaufgaben (Zachar und Strausz 2009).

Nach dem Intermezzo ${ }^{4}$ von 1918/1919 kam es nach der Wiederherstellung der bürgerlichen Rechtsordnung in der sogenannten Horthy-Ära (1919-1944) zur erneuten Verstärkung der Kammern, wobei auch in der Zwischenkriegszeit weiterhin der GA VI./1868 galt. Trotz der katastrophalen territorialen und wirtschaftlich-gesellschaftlichen Folgen des Friedensdiktates von Trianon (Vizi 2010, 2014; Koudela 2013) kam es in der Tätigkeit und der Struktur der CoC zu keiner großen Veränderung. Gleichzeitig versuchten sie jedoch, sich an die neue Situation anzupassen und intensivierten ihre Initiativen. Beispielsweise übernahmen sie eine bedeutende Rolle bei der Organisation der Internationalen Messe Budapest, der so genannten Ungarischen Woche, die jährlich veranstaltet wurde und die neuesten Ergebnisse des Handels und der Industrie dem breiteren Publikum vorstellte, des Weiteren bei der Unterstützung des Handelsschulennetzes, oder (in Partnerschaft mit dem Staat) bei der wirtschaftlichen Integration der mit den umstrittenen Wiener Schiedssprüchen von 1938 und 1940 Ungarn zugesprochenen Gebiete. Die CoC waren auch am kommunalen (städtischen und dörflichen), sowie staatlichen Handelsfachunterricht beteiligt. Die Kammern versuchten, die Entwicklung des Unterrichtsniveaus sowie die Probleme der pädagogischen Arbeit zu verfolgen und beteiligten sich durch mehrere konkrete Vorschläge zur Unterrichtsreform an der öffentlichen Debatte. Besonders die Handels- und Industriekammer Budapest war dafür bekannt, Schüler durch Stipendien zu unterstützen. Sie stellte auch einzelnen Institutionen, etwa Schulen, regelmäßig Unterstützungsgelder zur Verfügung. Zudem errichtete die CoC Budapest in den 1930er Jahren einen Fonds bei der Ungarischen Akademie der Wissenschaften, um die Autoren jener Studien zu belohnen, die Besonderes für die Popularisierung des Handels geleistet hatten (Strausz 2008). Wegen ihrer niveauvollen Arbeit waren die $\mathrm{CoC}$ angesehene Akteure des Gesellschaftslebens dieser Zeit, die auch im Oberhaus des ungarischen Parlamentes vertreten waren.

Mit der Besetzung Ungarns durch die Truppen des nationalsozialistischen Dritten Reiches am 19. März 1944 und der darauf folgenden Besetzung durch

\footnotetext{
${ }^{4}$ Die sozialistischen Regierungen unter Mihály Károlyi und Dénes Berinkey (1918) planten zwar starke Änderungen in der Struktur der $\mathrm{CoC}$, konnten diese aber nicht durchführen. Die Proletardiktatur unter der Leitung von Béla Kun (1919) verbot dann politische Parteien, Vereine und Kammern.
} 
sowjetische Truppen war eine Rückkehr zu den bürgerlichen Einrichtungen der vorherigen Epoche unmöglich. Von Anfang an war ein Streben nach dem Ausbau einer politischen, gesellschaftlichen und wirtschaftlichen Etablierung des sowjetischen Vorbilds zu verzeichnen, und dieses Modell lehnte im Zeichen der kraftvollen Zentralisierung jede Selbstverwaltungsorganisation strikt ab. Mit der wachsenden Verstaatlichung und der endgültigen Machtübernahme der Kommunisten bei den manipulierten Wahlen 1947 kam es dann zur Auflösung der traditionsreichen Kammerautonomie. Die bis dato den Kammern übertragenen Verwaltungsaufgaben wurden von Staatsorganen übernommen. Die Interessenvertretungstätigkeit wurde im Weiteren an andere Handels- und Industrievertretungen übergeben (Regierungsverordnung Nr. 5590/1948).

\section{Strukturwandel der ungarischen Handels- und Industriekammern nach der Wende}

Die ungarische Kammerbewegung erfuhr nach der politischen Wende 1989/1990 einen neuen Schub. In den nun folgenden zweieinhalb Jahrzehnten haben die ungarischen Wirtschaftskammern fast alle europäischen Kammermodelle in irgendeiner Form adaptiert; die von der Gesetzgebung festgelegten Wirkungsrahmen haben sich ständig geändert. Bis dato gibt es in Ungarn keinen einheitlichen politischen oder wissenschaftlichen Standpunkt zur Rolle und Bedeutung der Kammern in der Gesellschaft. Somit waren die Beziehungen der CoC zum Staat, ihre Aufgaben oder ihr Beitrag zur Gesellschaftsordnung ständig Veränderungen unterworfen - und sind es bis heute. Dies soll im Folgenden anhand von vier Punkten detaillierter dargelegt werden: Erstens sollen die gravierenden Veränderungen in der Regelung der Mitgliedschaft in den Kammern, zweitens der Wandel in den Dienstleistungen, drittens die Frage der Finanzierung und zuletzt die gemäßigteren Veränderungen in der internen Struktur der $\mathrm{CoC}$ dargestellt werden.

\subsection{Die Frage der Mitgliedschaft}

Parallel zur Wende kam es zur Reorganisierung und Wiederbelebung des traditionsreichen Kammersystems in Ungarn. Die entstehenden „Vereinskammern“, die ihre Rechtsgrundlage aus dem GA II. des Jahres 1989, dem sogenannten Vereinsgesetz ableiteten, hatten keine allzu engen Verbindungen zur Sphäre der Politik, in ihren Dienstleistungen waren sie sehr unterschiedlich ausgerichtet und zudem war auch ihre Fähigkeit beschränkt, Interessen durchzusetzen. Neben den klassischen 
CoC wurden zudem Kammern in zahlreichen Bereichen des Gesellschaftslebens gegründet: von den freien Berufen der Notare und Ärzte über die Jäger und Apotheker, bis hin zu Musikern sowie Sicherheits- und Wachdiensten.

Neben den sich als Kammern bezeichnenden Wirtschaftsorganisationen wurden auch andere, auf freiwilliger Mitgliedschaft beruhende Interessensvertretungsorganisationen gegründet: In diesem Zusammenhang sind die ebenfalls wiederentstehende Vereinigung der ungarischen Industriellen (GYOSZ), oder die Nationale Vereinigung des Kleinhandwerks und Kleinhandels (KISOSZ und KIOSZ) zu nennen. Durch die sehr ähnlichen Aufgaben, durch mehrmals sich überlappende Mitgliedschaften und durch den Mangel an politischer Unterstützung beziehungsweise das Fehlen von Startkapital konnte keine dieser Körperschaften effizient Dienstleistungen erbringen. Zudem prägten Zwiespalt und Rivalität die Beziehungen zwischen den Organisationen. Die strukturell ähnlichen Wirtschaftsverbände erhofften einen Mitgliederzustrom aus denselben Gesellschafts- und Wirtschaftsgruppen. Dies führte zu besonders brisanten Situationen und gleichsam einem „Legitimitätskrieg“ zwischen ihnen. An dieser Situation hatte auch die erste demokratisch gewählte Regierung unter József Antall (19901993) erheblichen Anteil. Da ein Großteil der Wirtschaftselite noch im staatssozialistischen System ihre Führungspositionen erlangt hatte, war die neue politische Macht ihr gegenüber zurückhaltend. Die Antall-Regierung wollte keine Akteure, die früher dem Staatssozialismus gedient hatten, in die politische Entscheidungsfindung einbeziehen. Die Regierung hat weder die Legitimität noch die Mitgliederrekrutierung noch die Selbstorganisation der Arbeitgeber- und Unternehmerorganisationen, besonders der $\mathrm{CoC}$, institutionell unterstützt. Obwohl die Kammern immer wieder die Schaffung eines zentralen Gesetzes über die Interessenvertretungen forderten, entschied die Regierung unter József Antall zuerst, dass ,sie ihre Hände nicht mit rechtlichen Mitteln in jener Hinsicht binden lassen will, mit wem, wann und auf welcher Grundlage verhandelt werden soll“" (Bruszt 1994, S. 225). Damit blieb die Mitgliederentwicklung der CoC in dieser Phase deutlich hinter den eigenen Erwartungen zurück.

Eine positive Veränderung für die $\mathrm{CoC}$ ergab sich infolge der Annäherung der ungarischen Regierung an die Europäische Union. Die Integration des Begriffes der „Körperschaft öffentlichen Rechts“ in das ungarische Rechtssystem war ein wichtiger Schritt bei der Angleichung des ungarischen Rechtssystems an westeuropäische Muster. Nach langen Diskussionen setzten sich jene Kammerverfechter durch, die ein an die traditionellen, öffentlich-rechtlichen Verwaltungsstrukturen angelehntes Kammersystem bevorzugten: Im Gesetzesartikel XCII. des Jahres 1993 wurden die Kammern als eine Art der Körperschaften öffentlichen Rechts genannt, wodurch Vereine, die in ihrem Namen den Begriff „Kammer“ führten, 
bis zum 31. Dezember 1995 verpflichtet wurden, ihren Namen zu ändern (Fazekas 2009, S. 94 ff.).

Existenz und Wirken der CoC wurden mit dem GA. XVI./1994 über die Wirtschaftskammern (Wirtschaftskammergesetz) auf eine neue, jedoch historischtraditionelle Grundlage gestellt. Die Regelung verwirklichte alle wesentlichen Merkmale eines modernen, demokratischen, aber historisch verwurzelten Interessenvertretungsmodells. Mit dieser Regelung von 1994 verlieh man den ungarischen $\mathrm{CoC}$ einen öffentlich-rechtlichen Status, Betroffenenmitwirkung und Staatsdistanz (Hendler 2005, S. 38). Dies bedeutete die erneute Einführung der Pflichtmitgliedschaft. Das klassische Legitimationsnarrativ begründete die Pflichtmitgliedschaft damit, dass nur dadurch die ,entsprechend effektive und ergebnisreiche“ Vertretung der „im Übergang begriffenen Wirtschaftsinteressen möglich werden kann und nur dadurch die Handelsverhältnisse, die Wirtschaftsethik stabilisiert, die Selbstverwaltungsmechanismen der Wirtschaft herausgebildet, die Information der Wirtschaftsakteure geschaffen und das internationale Handelsbeziehungssystem geformt werden könnte“. Den neuen Kammermitgliedern gegenüber wurde die Pflichtmitgliedschaft auch mit dem Gedanken der Selbstverwaltung begründet, demnach alle zur Interessenartikulierung beitragen konnten („Betroffenenmitwirkung“) und niemand außerhalb des Systems „nur als Leidtragender aber ohne Entscheidungsmitbestimmungsrecht" verblieb (GA. XVI./1994 Allgemeine Begründung III. 2). So gelänge es, die Einzelinteressen beziehungsweise die spezifischen Bestrebungen der einzelnen Sektoren und Wirtschaftszweige in den Hintergrund zu rücken beziehungsweise auszugleichen und in den Verhandlungen und Dialogprozessen mit der Regierung beziehungsweise mit der örtlichen Politik die Gesamtheit der ungarischen Wirtschaft zu vertreten (GA. XVI./1994, §§ 28, 31; GA. XVI./1994 Allgemeine Begründung III.). Damit legitimierte man eine Kollektivguterstellung in der Tradition zahlreicher westeuropäischer Staaten und zudem eine (vorläufige) Staatsentlastung.

Gleichwohl ist zu konstatieren, dass die Meinung der Kammermitglieder über die Pflichtmitgliedschaft von Anfang an sehr negativ ausfiel. Sie verstanden die Pflichtmitgliedschaft nicht als Verwirklichung von Demokratie und Selbstverwaltung, sondern eher als Last und als Rückkehr von Charakterzügen des Staatssozialismus. Dieses Gefühl wurde durch den „preußischen Charakter“ der Kammerverwaltung verschärft. Dies hatte zur Folge, dass die Mitgliederbeteiligung an den ersten Kammeraktionen besonders niederschmetternd ausfiel. Die Diskussion um die Pflichtmitgliedschaft wurde dadurch befördert, dass infolge einiger politisch motivierter Entscheidungen der neu amtierenden sozialistischliberalen Regierung von Ministerpräsident Gyula Horn (1994-1998) die Kammermitglieder unmittelbar mit Mitgliedsbeiträgen belastet wurden (siehe unten). 
Dies verstärkte den Widerstand der Mitgliedschaft erheblich: Der erste offizielle Kontakt der Kammer mit ihren Mitgliedern kam bei der Entrichtung der Pflichtbeiträge zustande.

Diese Stimmung führte dazu, dass die Politik sich erneut mit Kammerfragen befasste. Es schien ertragreich zu sein, die Frage nach den Erfahrungen mit den $\mathrm{CoC}$ auf die (wahl-)politische Agenda zu setzen; besonders mit Blick auf die Wählerklientel der kleinen und mittelständischen Unternehmen. So gab es bereits im Wahlkampf des Jahres 1998 zahlreiche Andeutungen seitens der damaligen Opposition (des nationalkonservativen FIDESZ und besonders der Kleinlandwirtspartei FKGP) über geplante Veränderungen der Kammerstrukturen, die dann nach dem Wahlsieg der Mitte-Rechts-Koalition auch durchgeführt wurden. Das in weiten Kreisen verbreitete Narrativ gegen die Pflichtmitgliedschaft beinhaltete den fehlenden Nutzen der Mitgliedschaft und der gezahlten Beiträge, die mangelnde Transparenz der Organisationspraktiken beziehungsweise, damit zusammenhängend, die geringe demokratische Qualität der Organisation. Damit war klar, dass der FIDESZ und seine Verbündeten hier Wahlstimmen und politische Unterstützung durch den Abbau protektionistischer Regulierungen erwarten konnten. Die erste Regierung von Viktor Orbán vertrat den Standpunkt, dass nur durch eine Reorganisation der $\mathrm{CoC}$, mit dem Ausbau eines neuen Interessenvertretungssystems und mit der Abschaffung der Pflichtmitgliedschaft den ungarischen KMUs geholfen werden könne: „Damit kommen wir den Ansprüchen der ungarischen Gesellschaft entgegen und die Unternehmen, die in die Kammer eintreten wollen, werden freiwillig, die geistigen Kapazitäten nutzend eine bessere, agilere, effektivere und dienstleistungsorientierte Kammer schaffen, als die gegenwärtige“ (Ungarisches Parlament 2003; Strausz und Zachar 2010).

Der nachfolgend verabschiedete Gesetzesartikel CXXI./1999 über die Wirtschaftskammern veränderte die Struktur des ungarischen Kammersystems grundlegend: Die Handwerkskammern wurden in die Handels- und Industriekammern integriert. Die Befugnisse der nunmehrigen Wirtschaftskammerstruktur wurden erheblich eingeschränkt. Die Kammern blieben zwar weiterhin öffentlich-rechtliche Körperschaften, aber ohne übertragene öffentliche Verwaltungsaufgaben. Zudem wurde die Pflichtmitgliedschaft aufgehoben. Dies hatte einen radikalen Rückgang der Mitgliedszahlen zufolge. Bei den $\mathrm{CoC}$ bedeutete dies, dass von den 727.384 registrierten Mitgliedern ab dem Oktober des Jahres 2000 lediglich 29.523 Unternehmen in der Kammer verblieben. Dabei handelte es sich durchweg um Großunternehmen, zumeist auch um Tochtergesellschaften oder Filialen von multi- oder transnationalen Firmen. Die ungarischen KMUs verließen die Kammer und traten auch keinem anderen Unternehmensverband bei. 
Auch aus diesem Grund war die wichtigste Frage nach der Jahrtausendwende die Rückgewinnung der Mitglieder, um als Organisation zu überleben. So definierten die $\mathrm{CoC}$ als ihren wichtigsten Zweck die Verbesserung des wettbewerblichen Marktumfelds (siehe unten). Somit war es das Ziel der Kammern, den Akteuren des Wirtschaftslebens mit belastbaren Wirtschaftsanalysen, Prognosen und Vorschlägen, mit dem Ausbau von zuverlässigen geschäftlichen Kontakten wie auch mit Rechts-, Steuer- und sonstiger Beratung möglichst wirksam zur Seite zu stehen. Aber trotz dieser Erweiterungen des Serviceangebotes verbesserte sich das Bild der $\mathrm{CoC}$ bei den Wirtschaftsunternehmen nur sehr langsam. Eine besondere Schwierigkeit ergab sich aus der paradoxen Rechtslage, dass die $\mathrm{CoC}$ ohne Pflichtmitgliedschaft weiterhin öffentlich-rechtliche Körperschaften, aber ohne übertragene öffentliche Verwaltungsaufgaben blieben und ihnen die „Wahrnehmung der Gesamtinteressen“ der Gewerbetreibenden vorgeschrieben wurde (GA. CXXI./1999, § 9). Deswegen traten die CoC bereits ab 2004 für eine Pflichtregistrierung der Unternehmen ein, da sie es als notwendig erachteten, über alle Marktteilnehmer informiert zu sein, um ihre öffentlichen Aufgaben effektiv ausführen zu können. Diese Pflichtregistrierung würde es auch erleichtern, den Rechtsfolgen der Ethikverfahren (Veröffentlichung einer Rüge oder Klärung) in der gesamten ungarischen Wirtschaft Geltung verschaffen zu können.

Aus aktuellen Umfragen und Recherchen ergibt sich, dass die CoC in ihren Bestrebungen, neue Dienstleistungssegmente zu mobilisieren und Mitglieder zu gewinnen, nicht allzu erfolgreich waren. Insgesamt stieg die Mitgliedszahl von anfänglich 29.000 bis 2004 auf fast 46.000 an, wobei dieser Kreis - infolge der Mitgliedschaft vor allem von multinationalen und Großunternehmen - nahezu zwei Drittel des ungarischen BIP abdeckte (Strausz und Zachar 2008). Dies ist wohl auch damit zu erklären, dass die KMUs in dieser Zeit keine allzu große Wirkung auf den Geschäftsbetrieb infolge der Kammerdienstleistungen verspürten.

Eine neue Debatte über die Kammern und die seit 2008 unter der sozialistischliberalen Regierung von Ferenc Gyurcsány eintretende Wirtschaftskrise führten jedoch dazu, dass erste gemeinsame Punkte mit den politischen Entscheidungsträgern gefunden werden konnten. Das Bedürfnis nach Verkleinerung und Entlastung der staatlichen Verwaltung und die Suche nach Antworten auf Fragen der Zeit (wie die Auswanderung der Jugendlichen) (Koudela 2011) sahen die CoC als Gelegenheit für die Übertragung neuer Aufgaben an. Nach Meinung der Kammerführungen könne es zu einer teilweisen Rückkehr zur früheren Position der wirtschaftlichen Selbstverwaltung als einer Selbstorganisierung der Betroffenen kommen. Diese Entwicklungen und wohl auch ein eingetretener personeller Wechsel in den Kammeradministrationen veränderte auch die bisher kritische 
Beziehung zu den CoC im politischen Mitte-Rechts-Lager. ${ }^{5}$ Ab Mitte 2009 zeigten Prognosen eindeutig, dass die größte Mitte-Rechts Oppositionspartei, die FIDESZ, die Parlamentswahlen 2010 mit großer Mehrheit für sich würde entscheiden können. So fiel die Aussage des Parteivorsitzenden Viktor Orbán, wonach die Pflichtmitgliedschaft wiederhergestellt und der Tätigkeitsbereich der CoC erweitert werden sollte, stark ins Gewicht.

Bereits im Februar 2011 begann man mit der gesetzesvorbereitenden Arbeit zur neuen Regulierung der Wirtschaftskammern. Diese wurde letztendlich nicht durch eine klassische Gesetzesänderung herbeigeführt. Stattdessen hat man im November 2011 eine eigentümliche und unkonventionelle Restrukturierung bei den $\mathrm{CoC}$ durchgeführt. In einem ,gemischten Gesetz“, das sich mit zahlreichen Steuerfragen beschäftigte, hat man das Kammergesetz von 1999 novelliert.

Nach der am 1. Januar 2012 in Kraft getretenen Änderung sind alle Einzelund Partnerschaftsunternehmen - mit Ausnahme der bereits unter die Zuständigkeit einer anderen Kammer fallenden - verpflichtet, sich bei der zuständigen $\mathrm{CoC}$ zu registrieren. Dazu müssen sie einen Beitrag von 5000 HUF (ca. 16 EUR) zur Erbringung der öffentlichen Kammeraufgaben bei der sie registrierenden Kammer entrichten. Dieser Beitrag gilt als öffentliche Schuld, die von der Landessteuerbehörde eingetrieben werden kann. Es ist wichtig, zu betonen, dass die Unternehmen mit der Registrierung nicht zu Kammermitgliedern werden. Die Mitgliedschaft bleibt weiterhin eine freiwillige. Für den geleisteten Beitrag können jedoch die im Gesetz festgelegten Dienstleistungen der Kammer in Anspruch genommen werden (siehe unten) (CLVI 2011, §§ 403-408).

Diese Modifikation hat weder die seit mehr als einem Jahrzehnt bestehenden Probleme der Wirtschaftskammern gelöst noch neue Möglichkeiten für die Unternehmen eröffnet. Aus deren Sicht müssen sie für die Kosten einer Organisation aufkommen, in der sie nicht Mitglied sind und von deren Dienstleistungen sie als „Nicht-Mitglieder“ kaum profitieren. Der Großteil der mit zahlreichen Steuern belasteten Unternehmen hat diese Veränderung nicht positiv aufgenommen: Eine besondere Belastung stellt selbst diese gering erscheinende Summe für die tausenden sogenannten Zwangsunternehmer dar. Zudem wird die Registrierung von Unternehmen bei den Kammern als unnütz bewertet, da die öffentlichen Register bislang online einsehbar sind.

\footnotetext{
${ }^{5}$ Dies wurde auch dadurch symbolisiert, dass man die Kontakte zum Präsidenten der Ungarischen Handels- und Industriekammer intensivierte und heute eine fast ,harmonisch“ zu nennende Beziehung besteht.
} 


\subsection{Die Dienstleistungen der Kammern}

Parallel zur und nach der politischen Wende versuchten sich die Vereinskammern mit freiwilliger Mitgliedschaft in erster Linie durch neue Dienstleistungen zu etablieren (Bennett i. d. B.). Diese erstreckten sich auf Bereiche, die in der staatssozialistischen Ära von keinem Arbeitgeber- oder Unternehmerverband wahrgenommen worden waren. Dies führte jedoch auch zu Konkurrenz: Neben den Wirtschaftsorganisationen, die sich als Kammern bezeichnen, wurden auch andere, auf freiwilliger Mitgliedschaft beruhende Interessensvertretungsorganisationen gegründet, welche dieselben Ziele vor Augen hatten. Die ebenfalls nach der Zwischenkriegszeit erneut entstehende Vereinigung der ungarischen Industriellen (GYOSZ) oder die Nationale Vereinigung des Kleinhandwerks und Kleinhandels (KISOSZ und KIOSZ) widmeten sich sehr ähnlichen Aufgaben. Zudem wurde der Ausbau der Kammerfunktionen auch dadurch erschwert, dass zum Teil aus Staatsbudgetmitteln, zum Teil durch Auslandsfinanzierung ,nach und nach neue Stiftungen, Büros, GmbHs gegründet wurden, die mit dem Ziel der Unternehmerunterstützung ganze Netzwerke ausbauen konnten“" (Farkas 2000, S. 64). In diesen wurden den Kammerdienstleistungen ähnliche Bestrebungen unternommen: Interessenvertretung, Wissenstransfer, Wirtschaftspartnersuche, Wirtschaftsförderung, Rechtshilfe.

Um diesen Trends standzuhalten, wurden von der größten IHK, der Handels- und Industriekammer von Budapest, im Jahre 1993 gleich zwei Unternehmerklubs ins Leben gerufen: der Business Club vereinigte Großunternehmen, während sich im Silver Club die klein- und mittelständischen Unternehmen assoziierten. Die dadurch begonnene Wirtschaftsförderung wurde zunehmend - später auch gesetzlich festgelegt - zum Tätigkeitsschwerpunkt der Kammern. Ebenfalls zu den Dienstleistungen für die Mitglieder gehörte die Herausgabe der Zeitschrift Budapest Business Journal und die Gründung mehrerer Stiftungen. Die Baross Gábor Vállalkozási Alapítvány (Stiftung Gábor Baross zur Unternehmensförderung) hatte das Ziel, die ungarischen KMUs auf die Herausforderungen der Marktwirtschaft und der Privatisierung vorzubereiten und ihnen Hilfe bei der Weiterentwicklung zu geben.

Neben diesen Dienstleistungsaufgaben versahen die Kammern jedoch auch traditionelle Aufgaben der Interessenvertretung und begannen bereits, Aufgaben der Zentralverwaltung zu übernehmen. So erhielten sie nach und nach die Befugnis zur Erteilung von Zeugnissen und Bescheinigungen, Ursprungszeugnissen und Zollpapieren von der zentralen Verwaltung. Sie waren an der Berufsbildung und dem Prüfungswesen durch Berufsprüfungskommissionen, Meisterprüfungen und verschiedene Berufsbildungsstipendien beteiligt. Sie übten eine weitreichende 
Gutachtertätigkeit aus (z. B. Begutachtung verschiedener Förderprojekte und fachlicher Preisausschreiben). Schließlich begannen die Kammern auch in speziellen, geregelten Fällen Sachverständige zu bestellen.

Eine Verbesserung ergab sich durch den neu eingeführten Status als öffentlichrechtliche Körperschaft und die Pflichtmitgliedschaft. Die Aufgaben der CoC wurden durch den Gesetzesartikel XVI./1994 über die Wirtschaftskammern in vier große Gebiete unterteilt. Neben der Vertretung der allgemeinen Interessen der Wirtschaft (Gesamtinteresse der Gewerbetreibenden) wurden den Kammern die traditionelle Wirtschaftsförderung, des Weiteren Sicherheitsgarantien für den Geschäftsverkehr (Pflege von Standesrecht und -sitte) und die Übernahme öffentlich-rechtlicher, gesetzlich festgelegter Aufgaben und Bereiche der Staatsverwaltung (Quasi-Behördenfunktion) vorgeschrieben (GA. XVI./1994, §§ 26-29). Zudem wirkten sie auch an der Besorgung von Staatsverwaltungsaufgaben hinsichtlich Wirtschaftsfragen mit. Bei der Vertretung der allgemeinen Interessen der Wirtschaft (Gesamtinteresse der Gewerbetreibenden) wurden den $\mathrm{CoC}$ in erster Linie statistische Aufgaben übertragen: Sie hatten unter ihren Mitgliedern Daten zu sammeln, diese zu analysieren und über die Ergebnisse sowohl die Öffentlichkeit als auch die Verwaltung zu unterrichten. Die CoC waren im Rahmen dieses Aufgabenbereiches berechtigt, bei Gesetzen, die das Funktionieren der Marktwirtschaft oder das Recht zur Unternehmensgründung beziehungsweise das Recht zur Freiheit des Wirtschaftswettbewerbs beschränken oder hemmen sollten, die Initiative zu ergreifen und die Modifikation dieser Rechtsnormen vorzuschlagen. Damit wurde die Konsultation der CoC bei Gesetzesvorlagen, die die Wirtschaft betrafen, unumgänglich.

Die Garantie der Sicherheit des Geschäftsverkehrs sowie der Pflege von Standesrecht und -sitte beinhaltete zahlreiche administrative Aufgaben (Ausstellung und Verifikation von Ursprungszeugnissen, Handelsdokumenten, usw., Zusammenstellung von Handelsbräuchen und -regeln usw.) wie auch die Aufstellung einer eigenen Datenbank (Handelsregister). Dies berechtigte die CoC gegen Mitglieder, die gegen das Interesse der Verbraucher oder gegen die Handelsethik verstießen, mit Sanktionen (Tadel, öffentliche Rüge) vorzugehen. ${ }^{6}$ Beim vierten Aufgabenkreis, der traditionellen Wirtschaftsförderung, sind die Unterstützung der Wirtschaftsinfrastruktur, die Förderung der technischen Entwicklung, die Betreuung der Außenhandelsbeziehungen, das Organisieren von Messen, die Beratung der Unternehmen, die Informierung der Mitglieder und die Bereitstellung von

\footnotetext{
${ }^{6}$ Schärfere Maßnahmen, wie z. B. die Suspendierung der Mitglieder oder Ausschluss aus der Kammer, konnten nicht ergriffen werden.
} 
Dienstleistungen zu finden. Damit ergab sich bei den CoC ein kulminierter Aufgabenkomplex, der traditionelle, historisch entstandene Aufgabenbereiche und neue, sich aus den Umständen der modernen Wirtschaft ergebende Aufgaben verband.

Neben den gesetzlichen Aufgaben waren die Wirtschaftskammern bemüht, mit neuen Dienstleistungen die Pflichtmitgliedschaft akzeptabel erscheinen zu lassen. Dies führte jedoch in dieser Periode der modernen Kammergeschichte zu weiteren Spannungen: Dadurch wurden die CoC selbst zu Marktteilnehmern und konkurrierten mit privaten Wirtschaftsteilnehmern, die ähnliche Dienstleistungen anboten. Die Dienstleistungen der $\mathrm{CoC}$ hatten als wichtigste Komponente die Bereitstellung von Leistungen für die Mitgliedschaft vor Augen. Als klassische Kammerdienstleistung kann in dieser Phase die Verlagerung der beruflichen Aus-, Um- und Fortbildung in gemeinnützige Wirtschaftsorganisationen angesehen werden. Den Erfordernissen des ungarischen Marktes entsprechend wurden in verschiedenen Branchen Aus- und Fortbildungskurse - besonders im Bereich des Managements und Marketings - durch gemeinnützige Firmen angeboten, die von der Kammer mitbegründet worden waren. Ebenfalls ist die Herausgabe der ab 1997 landesweit erscheinenden Wochenzeitschrift Üzleti 7 („Wirtschaftswoche“) als ein Erfolg zu werten. Sie ermöglichte es, die CoC-Stellungnahmen zu einzelnen geplanten Rechtsnormen, Ereignissen des Wirtschaftslebens und der Politik, zur Aus- und Fortbildung, zu Förderprojekten und auch dem EU-Beitritt in weiten Kreisen der Gewerbetreibenden bekannt zu machen.

Eine der wichtigsten Fragen dieses Jahrzehnts war die EU-Mitgliedschaft Ungarns. Das Aufgabenspektrum der Kammern wurde folgerichtig auch in diese Richtung ausgeweitet. Obwohl die hier wahrgenommenen Aufgaben wohl zum Bereich der Wirtschaftsförderung und Interessenwahrnehmung gezählt werden können, waren sie doch auch als Dienstleistungen für die Mitglieder anzusehen. Neben der Publikation einzelner Branchenregelungen in der EU wurden auch die Beratung bei EU-Förderprojekten, die Möglichkeit der europäischen Wirtschaftspartnersuche sowie die Unterstützungen bei internationalen Messen und Projekten immer stärker in den Vordergrund gestellt.

Infolge der Veränderungen des Rechtsrahmens 1999 waren die Kammern der gewerblichen Wirtschaft nachfolgend nicht nur interessiert, sondern in einem organisatorischen Überlebenskampf geradezu genötigt, sich nach dem Verlust eines bedeutenden Anteils ihrer Mitglieder (bis zu $95 \%$ !) neu zu profilieren und auch ihr Dienstleistungsangebot zu überdenken. Die Serviceleistungen stellten somit einen immer bedeutenderen Teil der Arbeit der $\mathrm{CoC}$ dar, zeigten aber wegen der geringen Mitgliedszahl nur beschränkt Wirkung.

Um auf die Fragen der Zeit reagieren zu können, wurde ein eigenes Büro für Handelsförderung etabliert: Hier wurde den Mitgliedern, oder auch denjenigen, 
die diese Dienstleistungen in Anspruch nehmen wollten, z. B. die Vermittlung von Unternehmenskontakten und Handelsberatung angeboten. Daneben konnten branchenspezifische Unternehmertreffen, Berufskonferenzen oder berufliche Weiterbildungen organisiert werden. Das Büro übernahm die Information über Rechtsnormveränderungen und bot Direktmarketing-Möglichkeiten an. Es wurden bei den $\mathrm{CoC}$ auch EU-Informationsbüros aufgestellt, die Beratung zu Fördermöglichkeiten sowohl zur EU als auch zu nationalen Programmen durchzuführen hatten.

Eine neue Dienstleistung war die Mediation. Es wurden eigene Abteilungen ins Leben gerufen, die versuchten in Einklang mit den allgemeinen Zielen und Aufgaben der Kammer alle Unternehmen und Fachleute zu adressieren, die in außergerichtlichen Konfliktlösungsverfahren geschäftliche und Alltagsstreitigkeiten beilegen wollen. Die ordentlich zahlenden Mitglieder können diese Dienstleistung so beziehungsweise mit besonderen Rabatten in Anspruch nehmen, während Nicht-Mitglieder Marktpreise entrichten.

Aktuell weisen die ungarischen $\mathrm{CoC}$ eine zweistufige Dienstleistungsstruktur auf. Mit der oben dargestellten neuen Pflichtregistrierung gibt es drei Grunddienstleistungen, die allen Unternehmen zur Verfügung stehen: Beratung in wirtschaftlichen, finanziellen, steuerlichen und Kredit-Fragen, Geschäftspartnersuche sowie Aufstellung von Datenbanken über mögliche Förderprojekte und Fördergelder. Die CoC Budapest hat für diese Aufgaben ein Büro für wirtschaftspolitische Fragen und Serviceleistungen aufgestellt, an das sich die registrierten Unternehmen seit August 2012 sowohl telefonisch als auch elektronisch oder sogar persönlich wenden können (Büro für wirtschaftspolitische Fragen und Serviceleistungen 2016). Den freiwilligen Vollmitgliedern werden weitere unentgeltliche oder ermäßigte Dienstleistungen angeboten. $\mathrm{Zu}$ diesen gehören etwa Rechtshilfe beim Arbeits- oder Sozialversicherungsrecht, Beratung bei Außenwirtschaftsfragen, Hilfe bei der Unternehmensgründung, Urkundenbeglaubigung, Teilnahme an Messen und Ausstellungen oder Ausgabe von Carnet A.T.A. Zolldokumenten (Ungarische Industrie- und Handelskammer 2012, IX, 47.2). Die neuen Dienstleistungen umfassen die Einführung einer einheitlichen Mitgliedskarte, die mit einem einheitlichen Kammer-Rabattsystem verbunden wurde. Dies bedeutet, dass ein bedeutender Teil der Kammerdienstleistungen mithilfe dieser Karte entweder unentgeltlich oder zu besonders günstigen Preisen bezogen werden kann. Zudem steht die neue Karte in Verbindung mit den Dienstleistungen des Euro Discount Club (EDC): die angebotenen Dienstleistungen und Waren können dadurch ebenfalls günstiger (mit einem Rabatt von 3-50\%) erworben werden (IHK-Pest 2016). Parallel mit der Einführung der neuen Mitgliedskarte wurde ein neues Kammerqualifikationssystem aufgestellt. Durch dieses Qualifikationssystem (KMR) können jene Unternehmen eine spezielle Handelsmarke 
erhalten, die den Vorschriften und Erwartungen der CoC entsprechen und dadurch als zuverlässige Firmen qualifiziert wurden. Eine solche Handelsmarke kann den Unternehmen beim Ausbau ihrer Wirtschaftsbeziehungen beziehungsweise bei Verhandlungen von Vorteil sein (IHK-Pest 2016).

Im Laufe der Neustrukturierung der Kammerdienstleistungen wurde eine Datenbank über Förderungen und Veranstaltungen aufgestellt. Diese elektronische Dienstleistung benachrichtigt automatisch die Unternehmen über neue Fördermöglichkeiten beziehungsweise Veranstaltungen und Weiterbildungen in den von ihnen angegebenen Themenbereichen. Damit parallel wurde eine B2B Plattform ins Leben gerufen: Die Wirtschaftsdatenbank Üzlet@Hálón ist unter anderem als Katalysator der ungarischen Wirtschaft gedacht und als virtueller Marktplatz eingerichtet, an dem nicht nur Unternehmerinformationen, Waren und Dienstleistungen, sondern auch Geschäftsangebote, Börsen und Messen sowie verschiedene Studien, Analysen und Wirtschaftsnachrichten den Firmen zur Verfügung stehen.

\subsection{Die Finanzierung der Kammern}

Die nach der politischen Wende konstituierten Vereinskammern der Industrie, des Handels und Gewerbes mit freiwilliger Mitgliedschaft erhielten für ihre Tätigkeiten keine staatlichen Finanzmittel. Sie waren zwischen 1989 und 1994 ohne übertragene öffentliche Aufgaben zum Interessenschutz ihrer Mitglieder und zur Verbesserung der Wirtschaftsverhältnisse tätig. Diese Ziele wurden jedoch auch von anderen Arbeitgeber- und Unternehmerorganisationen verfolgt. Dies hatte zur Folge, dass keine dieser Körperschaften über effektive Instrumente zur Durchsetzung ihrer Interessen verfügte. Zudem ist die Absicht erkennbar, dass die neuen Unternehmergemeinschaften von der Regierung bevorzugt unterstützt wurden. So gerieten die ,alten“ Institutionen, zu denen auch die CoC gehörten, in eine ungünstige Lage - besonders, weil sie noch oft durch die frühere sozialistische Elite geleitet wurden. Anders als in Polen (Kurczewska i. d. B.) erhielten sie aber im tripartitistischen sozialen Dialog einen sicheren Platz.

Die Finanzierung der öffentlich-rechtlichen Kammern mit Pflichtmitgliedschaft (1994-1999) gestaltete sich besonders interessant. Das neue Gesetz über die Wirtschaftskammern (GA XVI./1994) wurde ohne Gegenstimme im ungarischen Parlament verabschiedet und schuf somit die zweite umfassende Rechtsregelung der ungarischen Wirtschaftskammern nach 1868. Der Konsens über die wirtschaftlichen Grundlagen des neuen Kammersystems konnte leicht erzielt werden: Die CoC waren einhellig der Meinung, dass die finanziellen Grundlagen infolge der 
Verstaatlichung des Besitzes der historischen Kammerstrukturen durch das ,staatssozialistische System" nun mithilfe des Staates Ungarn wiederhergestellt werden müsse. Wegen der geplanten Pflichtmitgliedschaft bestand auch ein Konsens darüber, dass in den ersten zwei Jahren die Mitgliedsbeiträge ausgesetzt und später von der Steuer abgesetzt werden würden.

Dies bedeutete jedoch auch, dass die neuen $\mathrm{CoC}$, die in den Komitaten (den ungarischen Ländern) aufgestellt wurden, keine Rechtsnachfolger der früheren regionalen Vereinskammerorganisationen wurden, vor allem auch deswegen, weil sich aus einer früheren Regionalkammer im Jahre 1994 oft mehrere Komitatskammern konstituierten. Der Besitz der früheren Organisationen ging an die verschiedenen Arbeitgeberverbände, die weiterhin als Vereine ihre Interessenvertretungsarbeit versahen. Diese an sich schon schwierige Finanzsituation wurde durch diese politische Entscheidung verschärft. Die Regelungen des Kammergesetzes, die vom Konsens und der Zustimmung aller sechs Parlamentsparteien und der betroffenen Organisationen getragen waren, wurden schon im Jahre 1994 infolge eines Regierungswechsels grundlegend verändert. Mit einem zuvor nie gesehenen Manöver der ungarischen Politik hat die neue sozialistischliberale Regierung unter Gyula Horn nicht durch eine Novelle des Kammergesetzes, sondern mit dem Gesetz über das Budget des Jahres 1995 die finanziellen Regelungen der Interessenvertretungen umgestaltet. So blieben die Kammern ohne Finanzgrundlagen, sie erhielten weder Immobilien noch Geschäftsräume zur Verfügung gestellt und selbst die Pflichtbeiträge der Mitglieder mussten schon im ersten Jahr der Entstehung der Kammern entrichtet werden (Révész und Szakál 1994, S. 157).

Im Weiteren galten bei der Finanzierung der Kammern zwei Prinzipien: Einerseits hatte die Organisation aus den pflichtgemäß entrichteten Mitgliedsbeiträgen eine fixe Einnahmequelle; andererseits refinanzierte der ungarische Staat die von der Kammer übernommenen öffentlichen Aufgaben. Diese Einnahmen wurden von den Beträgen ergänzt, die durch Kammerdienstleistungen ins Budget flossen. Diese waren in der ersten Zeit nach Konstituierung der öffentlich-rechtlichen Kammern noch nicht allzu bedeutend, wuchsen aber durch die Ausweitung des Dienstleistungsangebots.

Mit dem Gesetzesartikel CXXI./1999 erfolgte die Aufhebung der Pflichtmitgliedschaft. Die Wirtschaftskammern blieben zwar weiterhin öffentlich-rechtliche Körperschaften, aber ohne übertragene öffentliche Verwaltungsaufgaben (siehe oben). Die Finanzierung der Kammerorganisationen wurde durch das Gesetz aus mehreren Einnahmequellen ermöglicht. Zu finden sind hier die Beiträge der Kammerzugehörigen, des Weiteren die Gebühren und Entgelte für die verschiedenen Dienstleistungen, die Einnahmen der kammereigenen Wirtschaftsunternehmen, 
zudem freiwillige Zuschüsse und die staatlichen Mittelzuweisungen. Mit dem Abbau der öffentlichen Aufgaben wurden den Kammern jedoch auch staatliche Finanzmittel entzogen. Dadurch und durch den drastischen Mitgliederrückgang erlitten die Kammern einen herben finanziellen Rückschlag. Diesem versuchten sie mit der Auflösung ihrer regionalen Büros, mit der Verringerung der Administration und mit neuen Einnahmen aus der Vermietung der leer gewordenen Büroflächen sowie mit neuen Dienstleistungssegmenten entgegenzuwirken. Dies führte jedoch in dieser Periode der modernen Kammergeschichte zu weiteren Spannungen, da sie zu Marktteilnehmern wurden (siehe oben). Dem Gesetz nach durften sich die Kammern nicht alleine zur Erzielung von zusätzlichen Einnahmen wirtschaftlich betätigen. Es war ihnen aber nicht verwehrt, bestehende oder neu geschaffene Infrastruktureinrichtungen optimal auszunutzen (,Randnutzung“).

\subsection{Die organisatorischen Strukturen der Kammern}

Trotz der zahlreichen gesetzlichen Veränderungen und dem wiederholten institutionellen Wandel in den vergangenen zweieinhalb Jahrzehnten veränderten sich die internen Strukturen kaum. Mit dem Gesetz von 1994 entstanden zuerst die vorläufigen Kammerorganisationskomitees. Mit der Einberufung der Delegiertenversammlungen, der Annahme der Satzungen und der Wahl der Kammerpräsidien und der leitenden Kammerbeamten entstanden die neuen funktionalen Gebietsselbstverwaltungsorganisationen. Die Dachorganisation, die Ungarische Handels- und Industriekammer (Magyar Kereskedelmi és Iparkamara, UIHK), die mit öffentlich-rechtlichem Status ausgestattet alle Gebietskammerorganisationen zusammenfasste, konnte ihre Arbeit erst nach der Konstituierung der Komitatskammern einleiten. Die 19 Komitate (Länder) Ungarns und die Hauptstadt Budapest entsandten aus ihren Kammern insgesamt 251 Delegierte in die konstituierende Versammlung der UIHK am 21. Dezember 1994. Danach wurde eine im Gesetz fixierte genaue Arbeitsteilung zwischen den Landesorganen und den Komitatsorganen durchgeführt. Im Aufgabenbereich der Landeskammer waren nationale Fragen sowie Fragen der internationalen Beziehungen, während sich die Komitatskammern vor allem um Fragen der örtlichen Wirtschaft und um die Mitglieder zu kümmern hatten. Die einzelnen Gebietskammern hatten ihre eigenen Statuten - im Einklang mit den Vorschriften des Gesetzestextes - zu schaffen. Darin konnten die einzelnen CoC selbst über die interne Struktur, über die zu schaffenden Sektionen, Abteilungen und Sparten entscheiden.

Wichtigstes Organ der Gebietskammern sind die Delegiertenversammlungen, die mindestens einmal jährlich zu Sitzungen zusammenzutreten haben. 
Hier werden die wichtigsten Funktionäre der Kammern (Präsident, Vizepräsidenten, Mitglieder des Präsidiums, Mitglieder des Kontrollausschusses, Mitglieder des Ethikausschusses) gewählt. Es wird des Weiteren über den Jahreshaushalt und die Selbstverwaltungsdokumente (Statuten, Geschäftsordnungen, etc.) entschieden. Die Delegiertenversammlung der Gebietskammern wählt auch ihre Vertreter für die Delegiertenversammlung der UHIK.

In der Zeit zwischen den einzelnen Delegiertenversammlungen versieht das Präsidium die Leitung der jeweiligen Kammer. Die Kammer kann gegenüber Dritten durch ihren Präsidenten wie auch, wenn dies im Statut festgelegt ist, durch Mitglieder des Präsidiums und durch den Sekretär vertreten werden. Die Arbeit der $\mathrm{CoC}$, der Jahreshaushalt und die rechtmäßige Tätigkeit der Organisation werden durch einen dreiköpfigen Kontrollausschuss überwacht. Eine Kontrollfunktion versieht ebenfalls der Ethikausschuss: Durch ihn wird die Einhaltung der Gewerberechtsnormen und des Urheberrechts überwacht; im Sinne der Ethiksatzung geht er gegen Wirtschaftsteilnehmer vor, die ihre Tätigkeit mit Verletzung der Verbraucherinteressen durchführen; er überwacht die Ethikregeln zum anständigen Marktverhalten und die Vorschriften hinsichtlich des Verbotes der Schmutzkonkurrenz. An der Spitze des administrativen Organs der Kammer steht der Sekretär, mit dem die Kammer ein Beschäftigungsverhältnis eingeht. Der Sekretär übt zudem über die Arbeitnehmer die Arbeitgeberrechte aus.

Neben diesen zur täglichen Arbeit notwendigen Strukturen können die $\mathrm{CoC}$ gemäß ihrer eigenen Statuten auch weitere Organe ins Leben rufen. Demnach gibt es in den ungarischen Gebietskammerorganisationen Sektionen und Fachkommissionen. Grundsätzlich kann in den einzelnen $\mathrm{CoC}$ festgestellt werden, dass gemäß ihrer Tätigkeitsbereiche drei große Sektionen aufgestellt worden sind: Sektion für Handel, Sektion für Industrie und Sektion für Handwerk. Die $\mathrm{CoC}$ Budapest und einzelne größere Komitatskammern haben zudem auch eine Sektion für Wirtschaftsdienstleistungen aufgestellt. Die Sektionen können sich in weitere Sparten gliedern, die die Mitglieder der Kammer gemäß ihrer Berufszweige, ihres Dienstleistungsspektrums und ihrer Handelstätigkeit gliedern. In der Landesdachorganisation, der UHIK, werden diese Sektionen und Sparten der verschiedenen Tätigkeitsbereiche durch Kollegien zusammengefasst. So gibt es unter anderem Kollegien für Industrie, Handel, Handwerk, Logistik, Innovation, Touristik, Fachbildung usw. Nach dem Beitritt Ungarns zur Europäischen Union wurde in der Landeskammer auch ein Kollegium für EU-Finanzierung ins Leben gerufen. 


\section{$4 \quad$ Fazit}

Abschließend ist festzuhalten, dass die $\mathrm{CoC}$ - nunmehr 25 Jahre nach der Wende keinen stabilen Platz im politischen und sozio-ökonomischen System Ungarns gefunden haben. Geprägt ist die Kammerfrage von den Turbulenzen, die im Anschluss an das ,sozialistische Erbe“ ein durchpolitisiertes Transformationsland prägen. Demgemäß sind die Entwicklungen bei weitem nicht abgeschlossen und der Tätigkeitsrahmen der $\mathrm{CoC}$ verändert sich weiterhin; vielleicht auch durch die erneute Einführung einer Pflichtmitgliedschaft.

Der Weg, der von den Vereinskammern über die öffentlich-rechtlichen Kammern mit Pflichtmitgliedschaft bis zur heutigen Situation der freiwilligen Mitgliedschaft mit Pflichtregistrierung beschritten wurde, war von zahlreichen Herausforderungen geprägt. Im Laufe dieser Zeit ist für die ungarischen Kammern ein institutioneller Wandel in Richtung eines leistungsorientierten Modells zu identifizieren, in dem das Portfolio der Dienstleistungen von erheblicher Bedeutung ist. Nachdem - infolge der geringen Mitgliedszahlen - eine eindeutige Input-Legitimation fehlt, war der Rechtfertigungsdruck immer stärker präsent. Die CoC waren in jeder Phase bestrebt, sich den neuen Rahmenbedingungen anzupassen und die ihnen gebotenen Möglichkeiten im Interesse ihrer Mitglieder und des Erhalts der eigenen Organisation zu nutzen. Sie haben erkannt, dass sich die wirtschaftlichen Kammerorganisationen im heutigen Ungarn tatsächlich in der Mitte zwischen Staatsverwaltung und fachlicher Selbstorganisation, im Verhältnis von Staatsmacht und Staatsbürger auf der Meso-Ebene ansiedeln (funktionale Selbstverwaltung) und sie den Herausforderungen nur durch eine Leistungsorientierung begegnen können. Derzeit versuchen sie im Rahmen der ihnen durch die nationale Gesetzgebung und durch die Richtlinien der EU vorgegebenen Möglichkeiten, alle Chancen für einen Anstieg der Mitgliederzahl und zur Versorgung des Handels, des Gewerbes und der Industrie mit spezifischen Serviceleistungen zu nutzen.

Der wiederholte institutionelle Wandel der CoC lässt diese als „Spielball der Politik" (Zachar und Strausz 2010) erscheinen. Das Auf und Ab in der Beziehung zwischen Politik und Wirtschaftskammern kann in erster Linie durch die unterschiedlichen Politikpräferenzen der Institutionen, die Differenzen zwischen den einzelnen Regierungen und den jeweiligen Kammervertretern und die Kalkulation der politischen Parteien mit Blick auf den Wählerstimmenmarkt (Unzufriedenheit der KMUs) erklärt werden (Strausz und Zachar 2010). Festhalten lässt sich, dass die Machtinhaber in jeder Periode nach der Wende bestrebt waren, darauf hinzuwirken, dass die Tätigkeit dieser Organisationen tatsächlich nur formal bleibe und ihre Interessenvertretungsarbeit auf ein Minimum beschränkt werde. 
Es ist zu konstatieren, dass die Mitgliedszahlen trotz der Pflichtregistrierung im Vergleich zur Zahl der KMUs nicht hoch erscheinen. Unter den Mitgliedern sind vor allem Großunternehmen zu finden, die durch die Kammermitgliedschaft effektive Vorteile auch im Bereich des Politischen erwarten.

$\mathrm{Zu}$ vermuten ist aber auch, dass diese politische Attitüde nicht langfristig aufrecht erhalten werden kann: Wegen des Glaubwürdigkeitsverlusts der ungarischen Politikerschicht und der oft an den Tag gelegten Inkompetenz wächst in der Gesellschaft das Verlangen nach Organisationen, die sich fachlich informiert und kompetent mit Fragen der Wirtschafts- und Sozialpolitik befassen. Die von den Kammern durchgeführte Interessensaggregation und (teilweise) -vertretung ist keine isolierte, für sich stehende Tätigkeit, sondern gliedert sich in den gesellschaftlichen Alltag ein. Wie zu Zeiten des klassischen Kapitalismus könnten CoC auch in einem Land im Übergang von sozialistischer Planwirtschaft zur sozialen Marktwirtschaft bedeutende Akteure sein. Meiner Meinung nach verrät es viel über die Betriebsmechanismen, ja sogar über die Auffassung von Demokratie eines politischen Systems, wie es sich gegenüber den Vertretern der nicht-politischen Sphäre verhält: Sieht es sie als Partner an und gewährt ihnen Zugang zu den Prozessen der Entscheidungsvorbereitung und -findung oder eben nicht? Und da die Kammern zu den erwähnten nicht-politischen Organisationen zählen, sind die Anerkennung oder die fehlende Anerkennung dieser durch den Staat beziehungsweise ihr Verhältnis zur Regierung gute Indikatoren dafür, wie die Politik des gegebenen Landes über die Herausforderungen und Methoden der Machtausübung im 21. Jahrhundert denkt. So wird die ungarische Politik hoffentlich bald gezwungen sein, einen ernsthaften Dialog und eine anhaltende Kooperation mit den Wirtschaftskammern zu eröffnen.

\section{Literatur}

Bruszt, L. 1994. Az Antall-kormány és a gazdasági érdekképviseletek. Kormány a mérlegen 1990-1994, hrsg. G. Csaba, H. Elemér, L. László, und G. Várnai, 208-230. Budapest: Korridor.

Büro für wirtschaftspolitische Fragen und Serviceleistungen. 2016. http://www.bkik.hu/ hir/3765-Gazdasagpolitikai-es-Szolgaltato-Iroda. Zugegriffen: 10. Januar 2016.

Farkas, G. 2000. Kamarák és vállalati érdekképviseletek az integrációs felkészülésben. Budapest: Osiris.

Fazekas, M. 2009. Balancierung zwischen dem öffentlich-rechtlichen und zivilrechtlichen Status. Neue Probleme in den Berufskammerregelungen. In Aktuelle Entwicklungen des Kammerwesens und der Interessenvertretung in Ungarn und Europa, hrsg. M. Dobák, J. Gergely, und W. Kluth, 94-96. Budapest, Halle: L'Harmattan. 
Gergely, J. 2006. Autonomien in Ungarn 1848-2000. Studienband. Budapest: L'Harmattan. Hendler, R. 2005. Geschichte und Idee der funktionalen Selbstverwaltung. In Handbuch des Kammerrechts, hrsg. W. Kluth, 23-39. Baden-Baden: Nomos.

IHK-Pest 2016. http://www.pmkik.hu/world/PMKIKWEB.nsf/all/Kamarai_tagkartya. Zugegriffen: 10. Januar 2016.

Kluth, W. 1997. Funktionale Selbstverwaltung. Verfassungsrechtlicher Status - verfassungsrechtlicher Schutz. Tübingen: Mohr Siebeck.

Kluth, W. 2005. Handbuch des Kammerrechts. Baden-Baden: Nomos.

Kluth, W. und F. Rieger. 2004. Grundbegriffe des Rechts der Industrie- und Handelskammern. Eine Darstellung nach Stichworten. Halle a. d. Saale: Institut für Kammerrecht.

Korinek, K. 1991. Staatsrechtliche Grundlagen der Kammer-Selbstverwaltung. Das Recht der Arbeit 41: 105-114.

Koudela, P. 2011. International migration from Central and Eastern European countries to Western Europe and the United States. Central European Political Science Review 12 (46): 67-88.

Koudela, P. 2013. Policy influencing society after trianon in Kassa-Kosice City. Central European Political Science Review 14 (51): 62-72.

Pelinka, A. und C. Smekal. 1996. Kammern auf dem Prüfstand. Vergleichende Analysen institutioneller Funktionsbedingungen. Wien: Signum Verlag.

Révész, P. und R. Szakál. 1994. Kézikönyv a gazdasági kamarákról. Budapest: Novorg.

Strausz, P. 2008. Kamarák a két világháború közötti Magyarországon. Budapest: L'Harmattan Kiadó.

Strausz, P. und P. K. Zachar. 2008. Gazdaságiés szakmai kamarák Magyarországon és az, Európai Unióban. Budapest: L’Harmattan.

Strausz, P. und P. K. Zachar. 2010. Die ungarischen Kammerstrukturen als Spielball der Politik? In Jahrbuch Recht und Ökonomik des Dritten Sektors 2009/2010 (RÖDS). Welche Aufsicht braucht der Dritte Sektor?, hrsg. H.-J. Schmidt-Trenz und R. Stober, 227256. Baden-Baden: Nomos.

Ungarische Industrie- und Handelskammer. 2012. Satzung in der Fassung vom 5. April 2012.

Ungarisches Parlament. 2003. T/5856. Über die Änderung des Gesetzesartikels CXXI./1999 über die Wirtschaftskammern. http://www.mkogy.hu/fotitkar/tvalk/tvalk.htm. Zugegriffen: 10. Januar 2016.

Vizi, László T. 2010. Ki legyen az aláíró? Vita a minisztertanácsban a trianoni békediktátum aláíróinak a személyéról. Közép-Európai Közlemények 3 (11): 109-132.

Vizi, László T. 2014. Ki írja alá a trianoni békét? Rubicon Történelmi Magazin 24 (260): 70-76.

Zachar, P. K. und P. Strausz. 2009. Die Autonomie- und Rechtsgeschichte des ungarischen Kammerwesens. Ein Abriss. In Jahrbuch des Kammer- und Berufsrechts 2008, hrsg. W. Kluth, 295-340. Halle a. d. Saale: Peter Junkermann. 